\title{
Corrections to MODIS Terra calibration and polarization trending derived from ocean color products
}

\author{
Gerhard Meister $^{a}$, Robert E. Eplee ${ }^{b}$, Bryan A. Franz $^{a}$ \\ ${ }^{a}$ NASA, Code 616, Goddard Space Flight Center, Greenbelt, MD 20771, USA; \\ ${ }^{b}$ Science Application International Cooperation, Beltsville, MD 20705, USA;
}

\begin{abstract}
Remotely sensed ocean color products require highly accurate top-of-atmosphere (TOA) radiances, on the order of $0.5 \%$ or better. Due to incidents both prelaunch and on-orbit, meeting this requirement has been a consistent problem for the MODIS instrument on the Terra satellite, especially in the later part of the mission. The NASA Ocean Biology Processing Group (OBPG) has developed an approach to correct the TOA radiances of MODIS Terra using spatially and temporally averaged ocean color products from other ocean color sensors (such as the SeaWiFS instrument on Orbview-2 or the MODIS instrument on the Aqua satellite). The latest results suggest that for MODIS Terra, both linear polarization parameters of the Mueller matrix are temporally evolving. A change to the functional form of the scan angle dependence improved the quality of the derived coefficients. Additionally, this paper demonstrates that simultaneously retrieving polarization and gain parameters improves the gain retrieval (versus retrieving the gain parameter only).
\end{abstract}

Keywords: remote sensing, scanners, on-orbit calibration, polarization

\section{INTRODUCTION}

Two copies of the Moderate-Resolution Imaging Spectroradiometer (MODIS) ${ }^{1}$ are currently in operation, providing top-of-atmosphere (TOA) radiances from 412nm to 14200nm. The first unit was launched in December 1999 on NASA's Earth Observing System (EOS) Terra satellite, the second on the Aqua satellite in May 2002. MODIS has 36 spectral bands on four different focal planes. The Ocean Biology Processing Group (OBPG) at NASA uses both instruments to produce ocean color data products ${ }^{2}$ and provides the data (approximately 23 Gigabyte per day for each instrument) to users around the world from the following website: http:oceancolor.gsfc.nasa.gov.

Each MODIS ocean color band has 10 independent detectors, and each of these detectors needs to be calibrated and characterized separately. Both MODIS instruments are calibrated using on-board calibrators ${ }^{3}$ and lunar irradiances. ${ }^{4}$ For MODIS Terra, these calibrations have not been sufficient to produce high quality ocean color products. ${ }^{5}$ The OBPG has developed a cross calibration method to improve the MODIS Terra characterization, using water-leaving radiances from another sensor as truth fields. ${ }^{6,7}$ This paper presents an update of the derived corrections relative to the MODIS Aqua instrument. Note that for the operational data processing, MODIS Terra ocean color products use SeaWiFS ${ }^{8}$ for the early part of the mission, and MODIS Aqua for the later part of the mission (SeaWiFS data is only available until 2010). The focus of this paper is the presentation of the temporal evolution of the MODIS Terra polarization sensitivity, which did not change significantly before the availability of MODIS Aqua data, so it was decided to use only MODIS Aqua data as truth data for this paper.

\section{STANDARD MODIS CALIBRATION AND CHARACTERIZATION METHODS}

The standard MODIS calibration equation ${ }^{9}$ uses the calibration coefficient $\mathrm{m}_{1}$ to describe changes in the radiometric sensitivity of the instrument. The $\mathrm{m}_{1}$ 's are used to derive earth scene reflectance $\rho_{E V}$ from the measured counts of the earth scene $\left(d n_{E V}\right)$ with:

$$
\rho_{E V} \cdot \cos \left(\theta_{E V}\right)=m_{1} \cdot d n_{E V}^{*} \cdot d_{E a r t h-S u n}^{2}
$$

Further author information:

G.M.: E-mail: Gerhard.Meister@nasa.gov 
where $\rho_{E V}$ is observed earth-viewing reflectance at a MODIS spectral band, $d n_{E V}^{*}$ is the temperature corrected measured counts, and $d_{E a r t h-S u n}$ is the distance between earth and sun, and $\theta_{E V}$ is the solar zenith angle. The scan angle dependence is corrected by dividing the measured counts by the RVS (response-versus-scan).

The calibration source for determining $\mathrm{m}_{1}$ on-orbit is the solar diffuser. The solar diffuser is viewed at an angle of incidence on the scan mirror of $50.3^{\circ}$. The calibration source for determining the RVS are lunar measurements through the space view port. For some bands only, measurements of selected earth view targets (several desert areas) are used for gain trending, see Sun et al. ${ }^{10}$

\section{VICARIOUS CALIBRATION METHOD}

The standard polarization correction equation for an uncalibrated instrument ${ }^{11}$ is

$$
L_{m}=M_{11} L_{t}+M_{12}\left(Q_{t} \cos 2 \alpha+U_{t} \sin 2 \alpha\right)+M_{13}\left(-Q_{t} \sin 2 \alpha+U_{t} \cos 2 \alpha\right)+M_{14} V_{t}
$$

where $\left(L_{t}, Q_{t}, U_{t}, V_{t}\right)$ is the Stokes vector at the TOA, $L_{m}$ is the measured radiance, $M_{11}$ to $M_{14}$ are the elements of the first line of the Mueller Matrix, and $\alpha$ is a rotation angle to adjust for different reference frames of the modeled atmospheric polarization and the instrument. Since $V_{t}$, the circular polarization component, is very close to zero at the TOA, $M_{14}$ is irrelevant. The polarization sensitivity parameters $M_{12}$ and $M_{13}$ were determined prelaunch at scan angles from $-45^{\circ}$ to $+45^{\circ}$, for each band, mirror side, and detector. ${ }^{12}$ The small variations of these parameters with detector were considered suspect and not applied in the ocean color processing. ${ }^{13}$ Note that $M_{11}=1.0$ means that eq. 1 (including the RVS corrections) was adequate, and $M_{12}=M_{13}=0$ means that there is no polarization sensitivity.

The cross calibration method has been described by Kwiatkowska et al., ${ }^{6}$ so here we provide only a brief summary. The correction parameters $\mathrm{M}_{11}, \mathrm{M}_{12}$, and $\mathrm{M}_{13}$ were calculated relative to the calibration (version 6.1.17.17_OC) provided by the MODIS Characterization Support Team (MCST). For a given day, the level 3 water-leaving radiances from MODIS Aqua are used to predict the top-of-atmosphere (TOA) radiances as seen by MODIS on that day, using the vicarious calibration technique described in Franz et al., $2007,{ }^{14}$ and the atmospheric correction model consistent with the R2010.0 reprocessing. Thus, all relevant components of the Stokes vector $\left(L_{t}, Q_{t}, U_{t}\right)$ can be modeled. This allows the estimation of the radiometric calibration parameters $\mathrm{M}_{11}$ as well as the polarization sensitivity parameters $\mathrm{M}_{12}$ and $\mathrm{M}_{13}$ by minimizing differences between the left-hand and right-hand side of eq.2 over many observations.

The parameters $\mathrm{M}_{11}, \mathrm{M}_{12}$, and $\mathrm{M}_{13}$ are derived simultaneously by constructing a matrix of thousands of instances of eq. 2, each line corresponding to a matchup of one MODIS Terra measurement $\left(L_{m}\right)$ with one predicted TOA Stokes vector.

In the correction process, $\mathrm{M}_{11}$ is applied first, i.e. $\mathrm{M}_{12}$ and $\mathrm{M}_{13}$ need to be applied relative to a calibrated instrument. The polarization correction equation for a calibrated instrument ${ }^{11}$ is thus

$$
L_{m}=L_{t}+m_{12}\left(Q_{t} \cos 2 \alpha+U_{t} \sin 2 \alpha\right)+m_{13}\left(-Q_{t} \sin 2 \alpha+U_{t} \cos 2 \alpha\right)+m_{14} V_{t}
$$

with

$$
\begin{aligned}
& m_{12}=\frac{M_{12}}{M_{11}} \\
& m_{13}=\frac{M_{13}}{M_{11}}
\end{aligned}
$$

The modeled TOA radiances are compared to the radiances measured by MODIS for every scan angle, mirror side, and detector. This means that the instrument characterization parameters $\left(\mathrm{M}_{11}, \mathrm{~m}_{12}, \mathrm{~m}_{13}\right)$ can be derived as a function of scan angle, mirror side, and detector. To reduce noise, the scan angle dependence is modeled by a fourth order polynomial for $\mathrm{M}_{11}$, and as a quadratic function for $\mathrm{m}_{12}$ and $\mathrm{m}_{13}$.

The method assumes that the near-infrared (NIR) bands do not need a correction. Fig. 1 shows that the globally averaged aerosol optical thickness (AOT) ocean color products have very similar temporal trends for MODIS Terra and MODIS Aqua, which suggests that the MCST calibration of the MODIS NIR bands indeed 

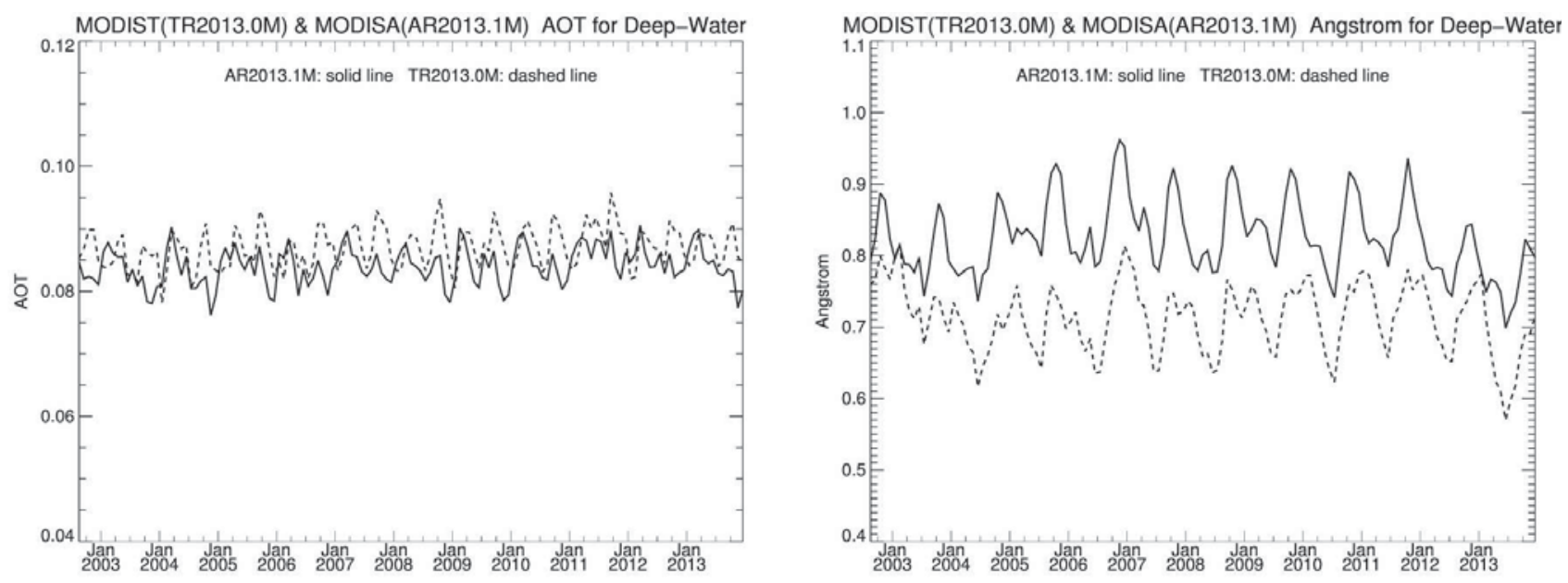

Figure 1. Globally averaged MODIS AOT (left) and angstrom (right), for MODIS Aqua (solid line) and MODIS Terra (dashed line). The methodology to create these plots is explained in Franz et al. ${ }^{5}$

does not need a correction. The Angstrom trends for Terra and Aqua MODIS are generally in good agreement, but with some bias likely associated with the vicarious calibration. The fact that both the AOT and Angstrom trends remain in good agreement after 2005, when the estimated polarization changes in MODIS Terra become significant, suggests that the polarization change detected in the visible bands is not an artifact of the NIR calibration.

The retrieval of the instrument characterization parameters is repeated for one day in every month of the mission. This results in a time series for the instrument characterization parameters. The parameters are retrieved as a function of scan angle for each day. Examples for $M_{11}, m_{12}$ and $m_{13}$ of band 8 (412nm) are shown below in Figs. 2, 4 and 5, respectively, to give an estimate of the noise in the measurements.

In Fig. 3, $M_{11}$ were retrieved while keeping $m_{12}$ and $m_{13}$ fixed at their prelaunch values. It can be seen that at the high scan angles, not including the polarization parameters in the retrieval more than doubles the amplitude of the seasonal oscillation for the $M_{11}$. Furthermore, the long term trends have changed significantly (e.g. for frame 1250 at the end of mission, the $M_{11}$ in Fig. 2 are at about 1.1, versus only about 1.05 for Fig. 3. A difference of $5 \%$ in the gain calibration is highly significant, not only for ocean color applications.

The MCST approach does not take into account a changing polarization sensitivity. Neither do the results shown in Fig. 3. As expected, the $M_{11}$ in Fig. 3 are closer to 1.0 than the $M_{11}$ in Fig. 2. The large deviations of the $M_{11}$ from 1.0 are therefore likely a result of the fact that the MCST calibration approach does not take into account polarization sensitivity, and not an indication that there is systematic problem with their approach for unpolarized radiances.

A. Lyaspustin (private communication) reported a potential overcorrection at the beginning of the scan when applying the operational OBPG correction coefficients to MODIS Terra land products (see also Lyapustin ${ }^{15}$ ). We discovered that the reason was that the scan angle dependence for the $m_{12}$ coefficients was modelled as linear, but a quadratic function should have been used. The large increase of polarization sensitivity at the end of scan produced a large slope of the linear function, which lead to $m_{12}$ values at the beginning of the scan that were too low, see Fig. 2 in Meister et al. ${ }^{7}$ (the $m_{12}$ decrease to about -0.1 after 2007 for band 8 for the beginning of the scan). The data presented here use a quadratic function to fit the scan angle dependence of $m_{12}$ and $m_{13}$. As can be seen in Fig. 4, the large increase in $m_{12}$ at the end of scan (frame 1250) no longer produces a decrease of the $m_{12}$ at the beginning of scan (frame 22). In effect, there is now a small increase of $m_{12}$ for frame 22 after 2007. 

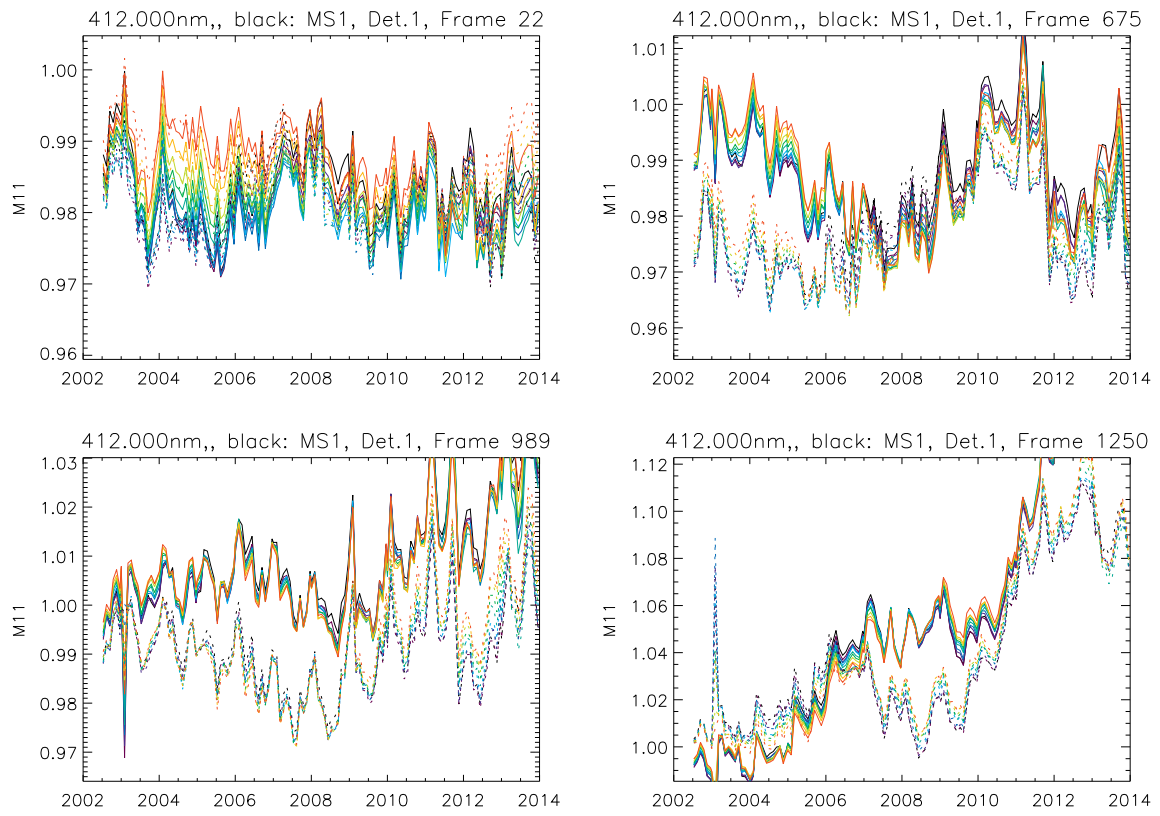

Figure 2. $\mathrm{M}_{11}$ for mirror sides 1 (solid line) and 2 (dashed line), detector 1-10 (black to red) of MODIS Terra band 8 $(412 \mathrm{~nm})$ as a function of time at four different scan angles (see plot title). All parameteres $\left(M_{11}, m_{12}\right.$ and $\left.m_{13}\right)$ were retrieved simultaneously. Note the different ordinate (y-axis) for each plot.
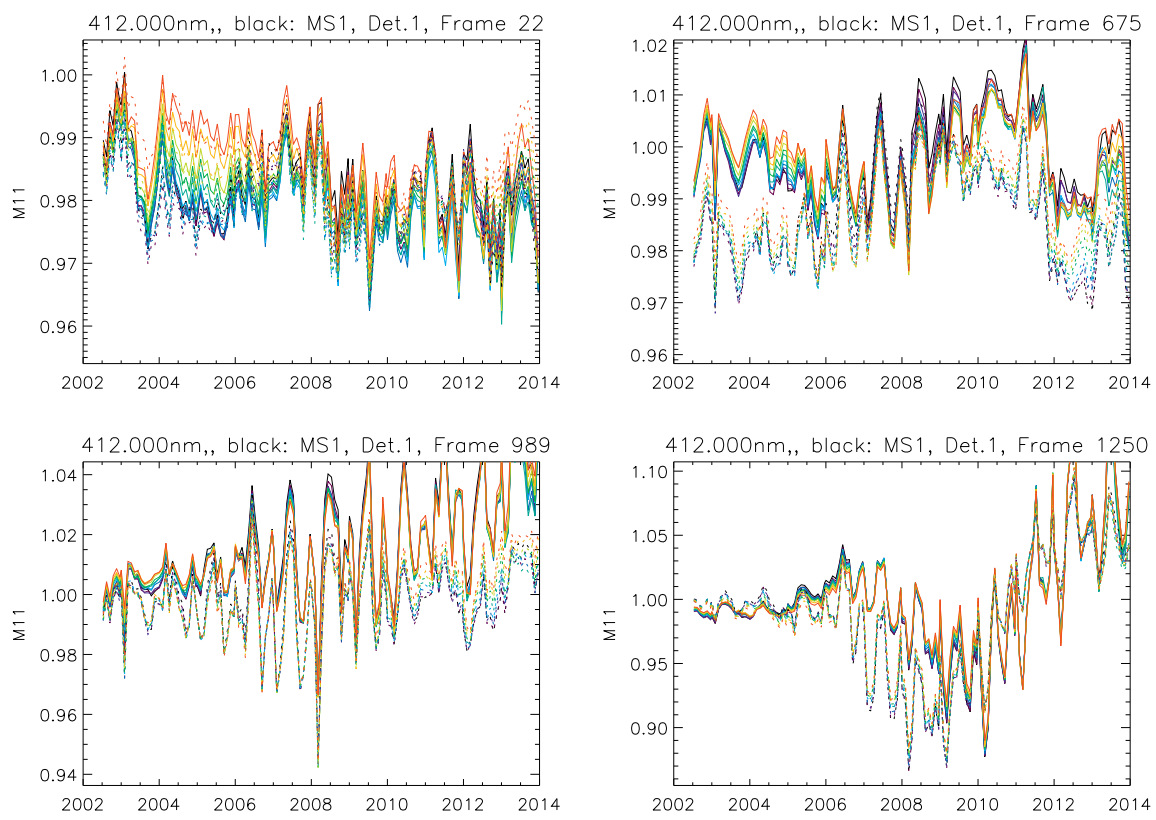

Figure 3. $\mathrm{M}_{11}$ for mirror sides 1 (solid line) and 2 (dashed line), detectors 1-10 (black to red) of MODIS Terra as a function of time at four different scan angles (see plot title). Only $M_{11}$ was retrieved, $m_{12}$ and $m_{13}$ were fixed at their prelaunch values to demonstrate that retrieving both gain and polarization paramters simultaneously improves the gain retrievals. 

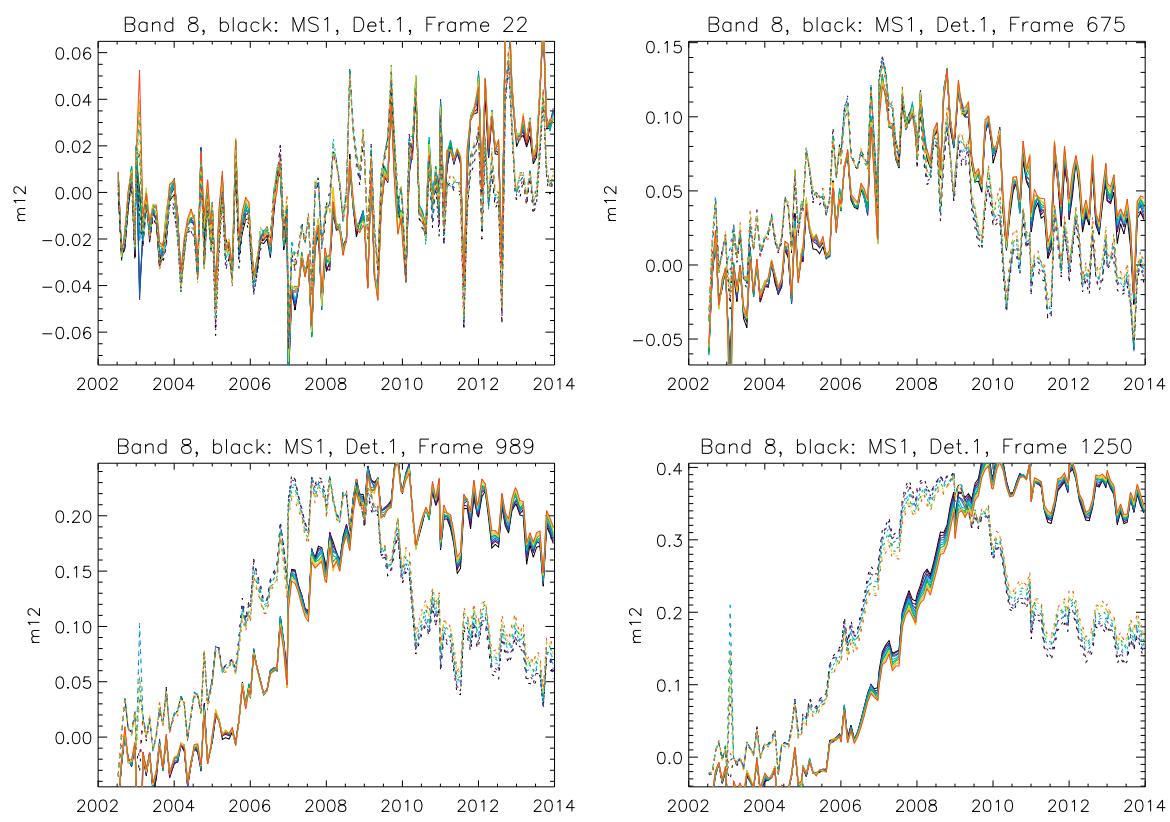

Figure 4. $\mathrm{m}_{12}$ for mirror sides 1 (solid line) and 2 (dashed line), detector 1-10 (black to red) of MODIS Terra band 8 $(412 \mathrm{~nm})$ as a function of time at four different scan angles (see plot title). All parameteres $\left(M_{11}, m_{12}\right.$ and $\left.m_{13}\right)$ were retrieved simultaneously. Note the different ordinate (y-axis) for each plot.
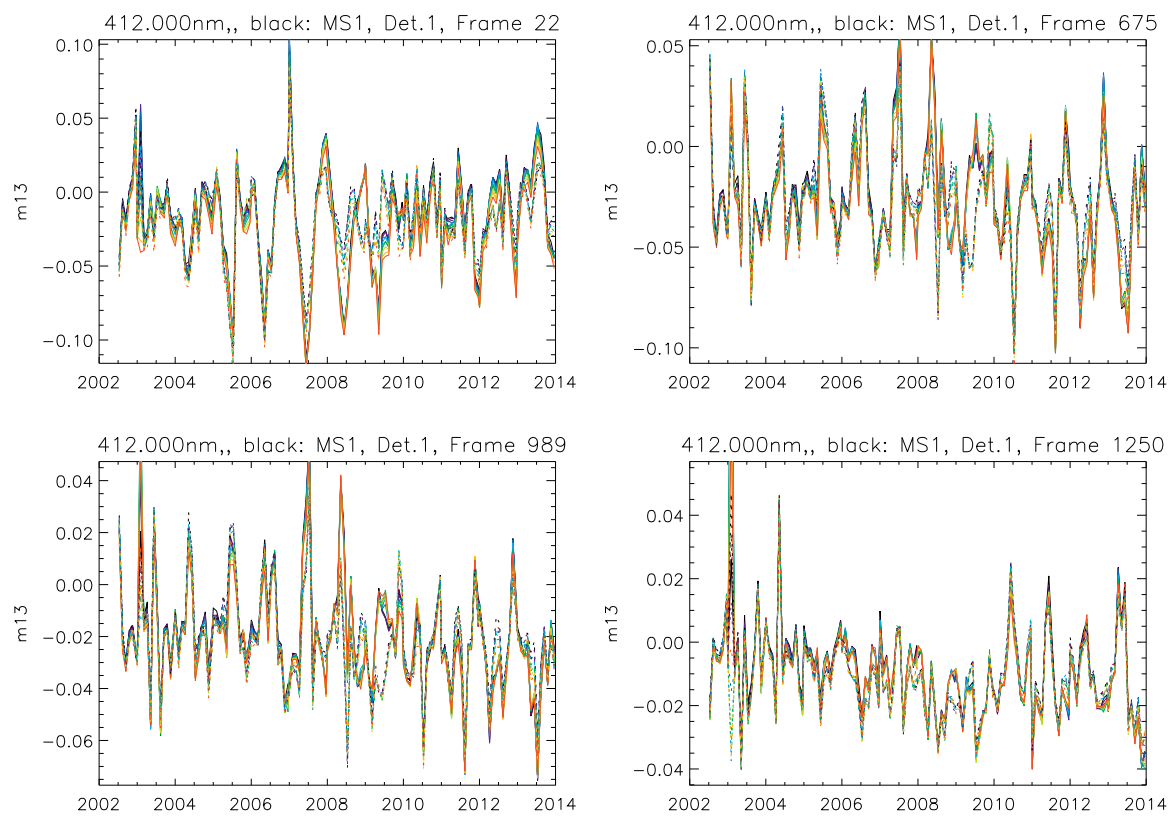

Figure 5. $\mathrm{m}_{13}$ for mirror sides 1 (solid line) and 2 (dashed line), detector 1-10 (black to red) of MODIS Terra band 8 $(412 \mathrm{~nm})$ as a function of time at four different scan angles (see plot title). All parameteres $\left(M_{11}, m_{12}\right.$ and $\left.m_{13}\right)$ were retrieved simultaneously. Note the different ordinate (y-axis) for each plot. 


\section{RESULTS AFTER TEMPORAL SMOOTHING}

It can be seen in Figs. 2, 4 and 5 that there is significant noise in the retrievals. To reduce noise, a running 1 year median is calculated for 15 different scan angles (for equally spaced scan angle intervals), and the polynomial as a function of scan angle is recalculated for the median values for each date. To further reduce noise, a temporal smoothing filter is applied ( \pm 2 months smoothing). This method was consistenly applied to all bands and all three parameters $\left(M_{11}, m_{12}\right.$ and $\left.m_{13}\right)$. Previously, (see e.g. Meister et al. ${ }^{7}$ ) the $m_{13}$ were kept constant at their prelaunch values. The additional amount of data available since the latest analysis gave us the confidence to trend the $m_{13}$ as well.

An overview of the results for mirror side 1, detector 5, is given in Figs. 6 to 8 . The shortest wavelengths show the largest values, and the end of scan varies much more than the beginning of the scan for most bands. These effects are expected, because the shortest wavelengths have always been more challenging to calibrate for the MODIS instruments, and the end of scan has the largest angle of incidence on the scan mirror, which favors a stronger polarization sensitivity. For band $10(488 \mathrm{~nm})$, the largest correction for $M_{11}$ is actually in the center of the scan. Note that the results for $748 \mathrm{~nm}$ are just a consistency check: the NIR bands are assumed to be perfectly calibrated by our approach, i.e. the $M_{11}$ should be close to 1.0 and the $m_{12}$ and $m_{13}$ should be close to their prelaunch values; for band 16 ( $869 \mathrm{~nm}$, not shown here) the results are even better than for band 15 $(748 \mathrm{~nm})$.

For almost all bands, the magnitude of the parameters increases with time. This is not surprising, the more the instruments (both MODIS Aqua and Terra) age, the larger correction coefficients we expect.

The results for all detectors and both mirror sides at the end of scan are shown in Figs. 9 to 11 . Fig. 9 shows the result for $M_{11}$. It can be seen that especially for the shorter wavelengths, there is a clear mirror side difference. For the longer wavelengths (555nm to $678 \mathrm{~nm}$ ), the mirror sides agree quite well, but one detector (detector 1 , black line) is an outlier. In general, shorter wavelengths show larger values (up to almost 1.2 for $412 \mathrm{~nm}$ and $443 \mathrm{~nm})$. The red MODIS ocean bands $(667 \mathrm{~nm}$ and $678 \mathrm{~nm})$ show very good agreement between MODIS Aqua and Terra (within $\pm 1 \%$ ), but the red MODIS Terra land band $(645 \mathrm{~nm})$ shows an increase of $M_{11}$ of about $5 \%$ (there is a similar increase at $859 \mathrm{~nm}$ ). The relatively large difference between MODIS Terra and MODIS Aqua at $645 \mathrm{~nm}$ (and $859 \mathrm{~nm}$ ) is rather surprising, it does not appear in independent trend analysis by MCST of those bands at high radiances, but was confirmed by MCST for ocean radiances (private communication, Amit Angal, MCST). This could mean that the linearity of those bands is changing with time.

Fig. 10 shows the result for $m_{12}$. It can be seen that the shortest wavelength bands had a very large increase in polarization sensitivity over time after 2005. Mirror side 2 starts to increase earlier than mirror side 1 . At $412 \mathrm{~nm}, m_{12}$ of mirror side 2 reaches a maximum of about 0.4 and then decreases. No other band shows a similar behavior. The 645nm band has almost no change in polarization sensitivity over time, all other bands change.

Fig. 11 shows that not only band 8 (412nm) shows a decrease of $m_{13}$, but also some longer wavelengths, especially bands 13 and $14(667 \mathrm{~nm}$ and $678 \mathrm{~nm})$. The amount of the decrease is up to 0.02 .

Note that some of the plots in Figs. 9 to 11 show short term variations that may or may not be valid changes of the MODIS Terra characteristics. For a reprocessing of the MODIS Terra data, the OBPG may decide to apply further temporal smoothing before applying corrections to the MODIS Terra data.

\section{CONCLUSIONS}

Recently, there has been increased interest from other disciplines (land, atmosphere) in the MODIS Terra polarization correction, and several studies $\left({ }^{15-17}\right)$ have reported increased product quality using the corrections derived by the OBPG. This paper provides a reanalysis of the correction factors for MODIS Terra and extends the time period covered through 2013. Note that the OBPG expects to reprocess the truth field used in this analysis (the MODIS Aqua data record) in the near future. Therefore, the coefficients presented here are only preliminary, but they do provide insight into the expected changes.

Extending the time period showed that the polarization correction parameter $m_{13}$ is not constant, as was assumed previously. ${ }^{6,7}$ The application of this change has not yet been tested in the correction of ocean color products, but it will be in the near future. 

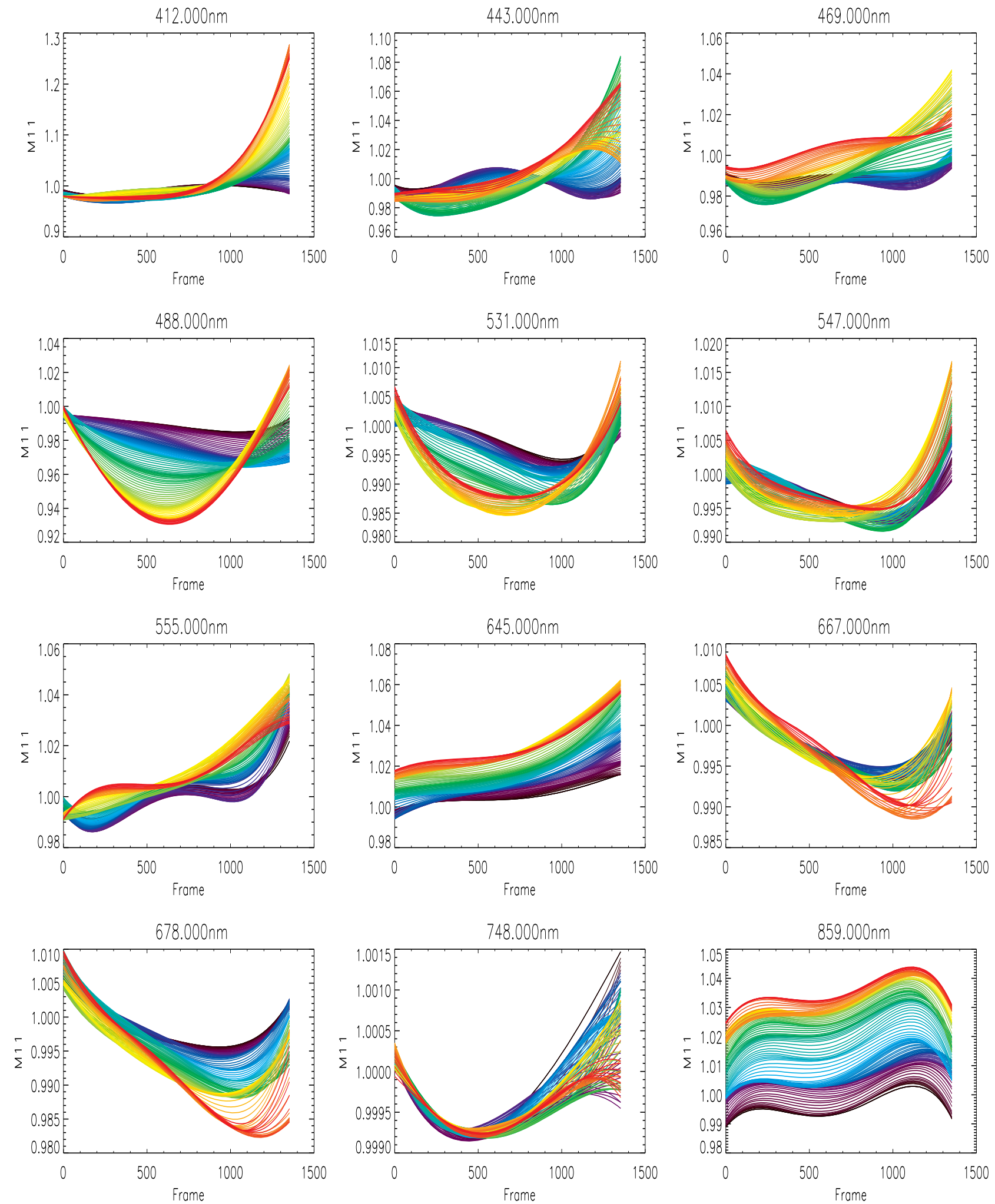

Figure $6 . \mathrm{M}_{11}$ for mirror side 1, detector 5 of MODIS Terra as a function of scan angle. Colors indicate time (black=2002, red $=2013$ ). Note the variation of the ordinate axis among the plots. 

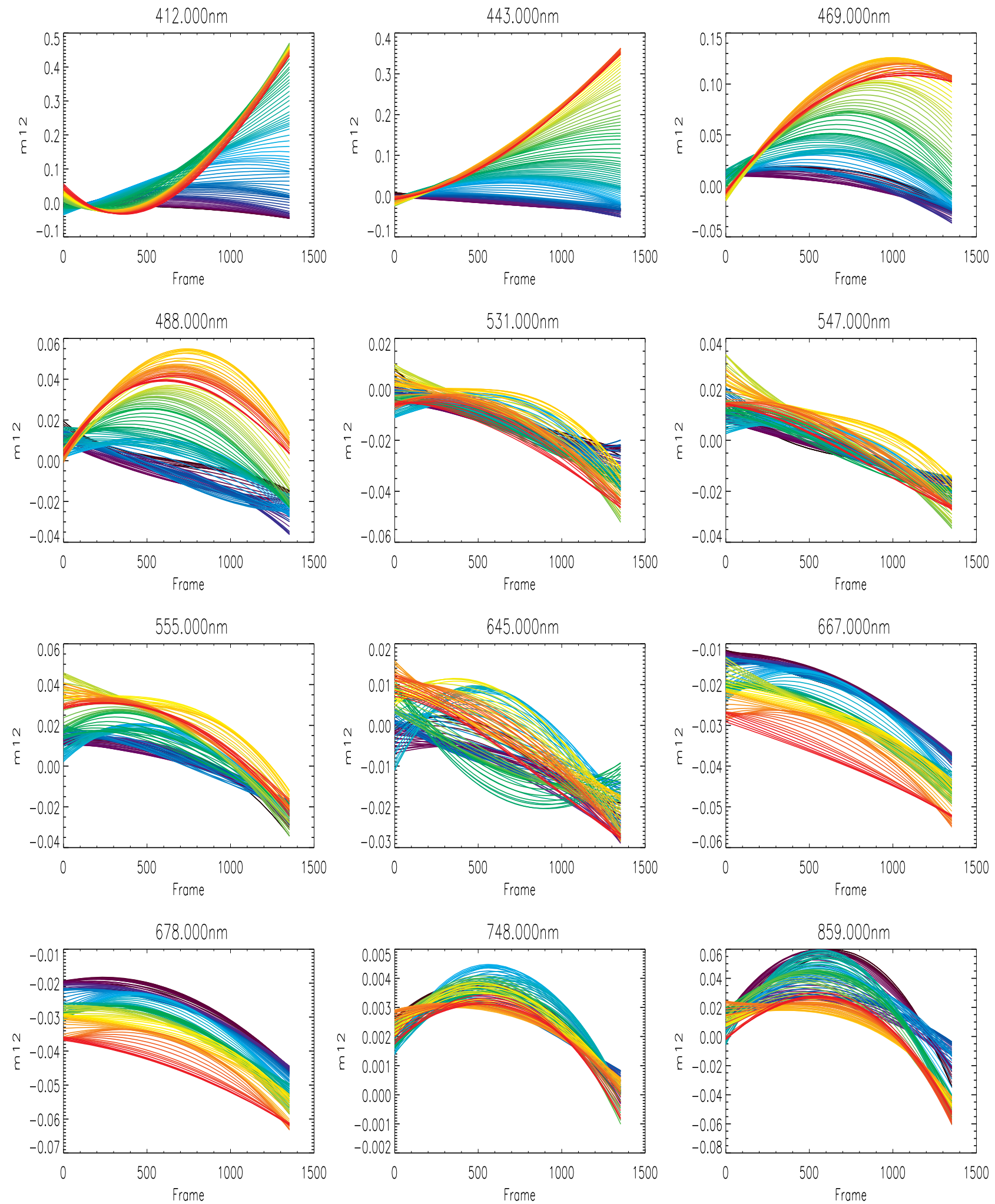

Figure 7. $\mathrm{m}_{12}$ for mirror side 1, detector 5 of MODIS Terra as a function of scan angle. Colors indicate time (black=2002, red $=2013)$. Note the variation of the ordinate axis among the plots. 

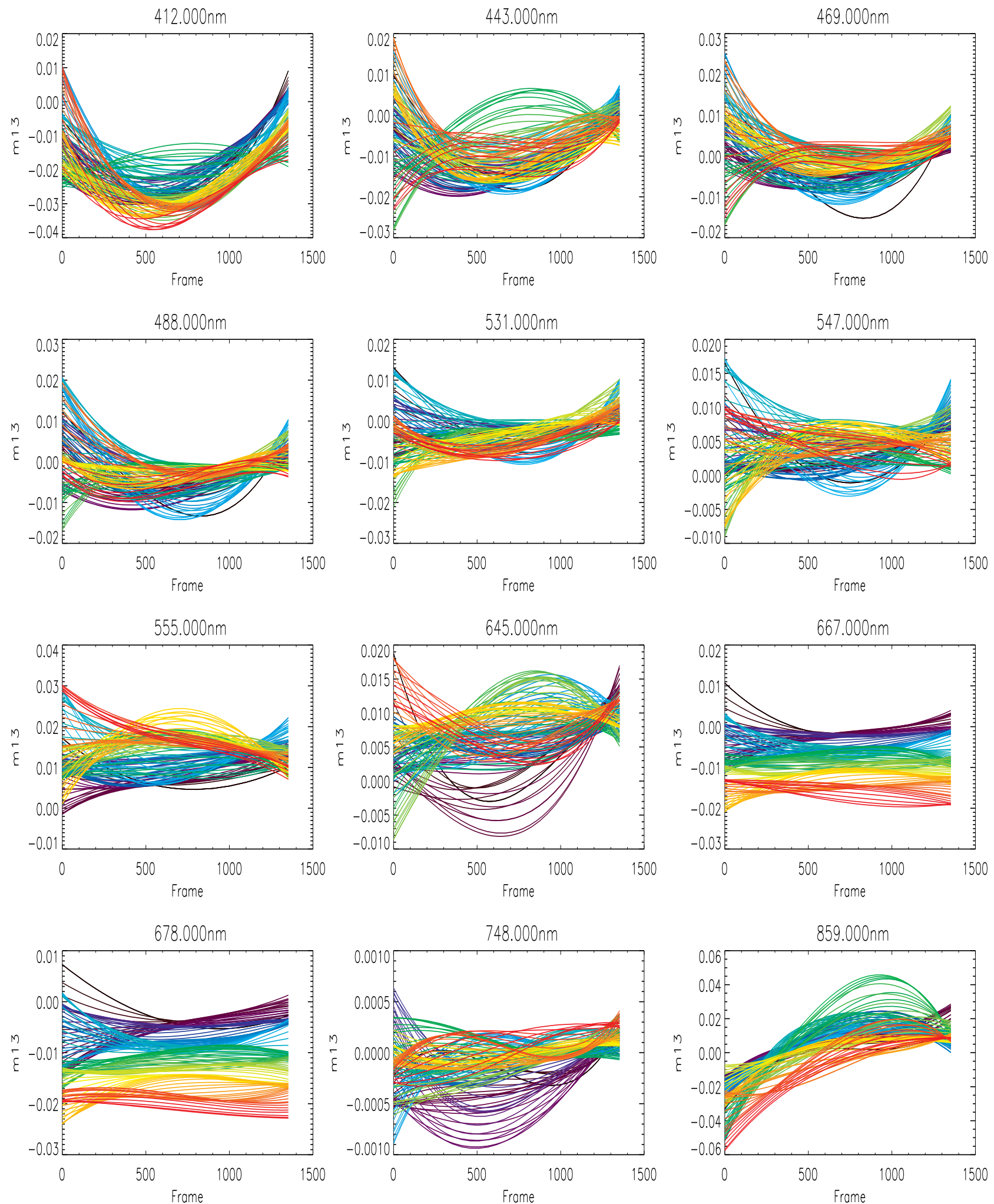

Figure $8 . \mathrm{m}_{13}$ for mirror side 1 , detector 5 of MODIS Terra as a function of scan angle. Colors indicate time (black=2002, red $=2013$ ). 


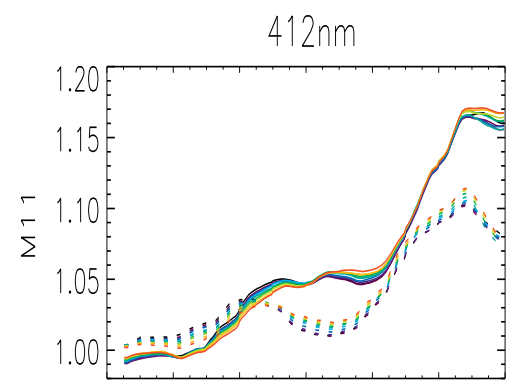

2002200420062008201020122014

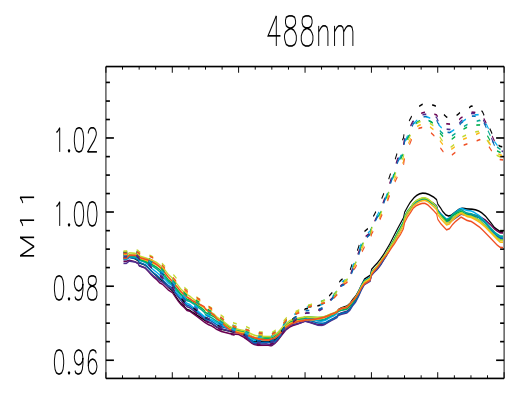

2002200420062008201020122014

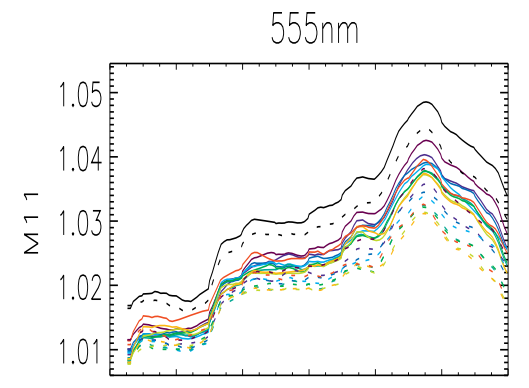

2002200420062008201020122014

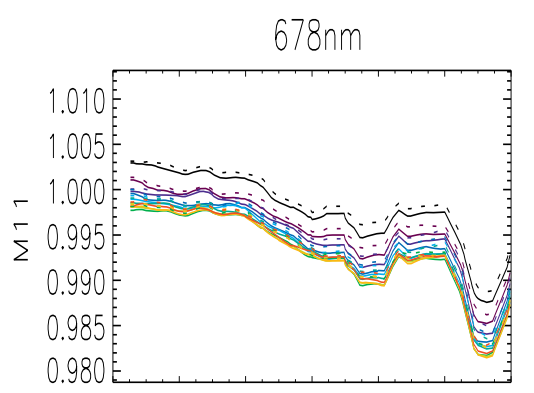

2002200420062008201020122014

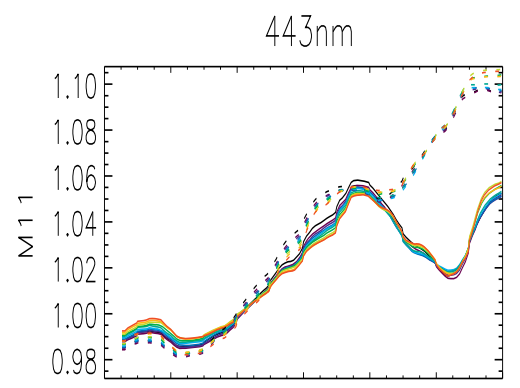

2002200420062008201020122014

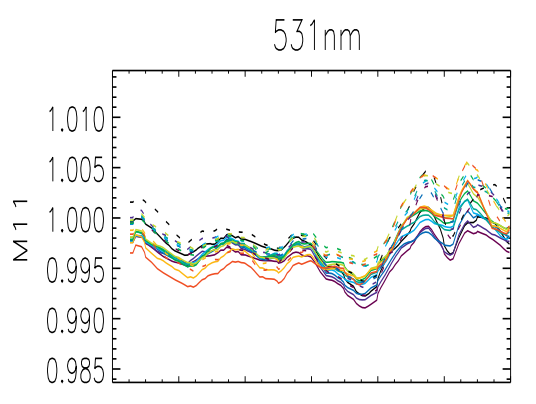

2002200420062008201020122014

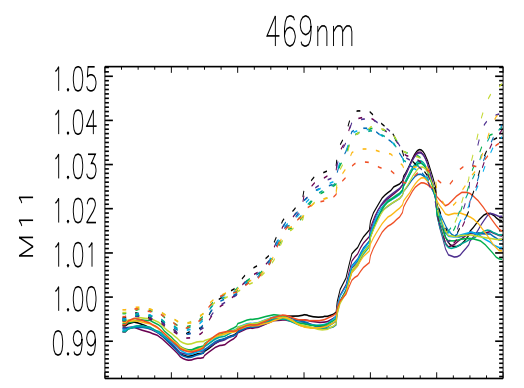

2002200420062008201020122014

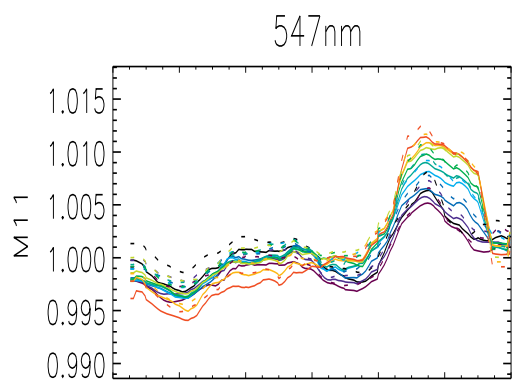

2002200420062008201020122014

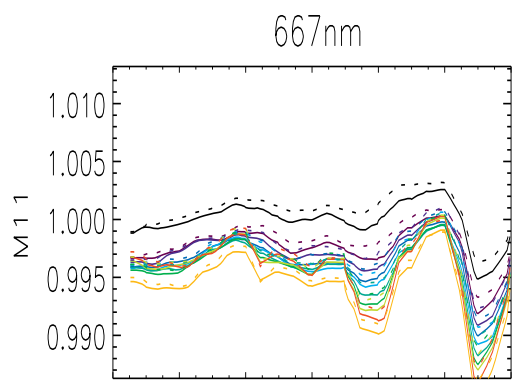

2002200420062008201020122014

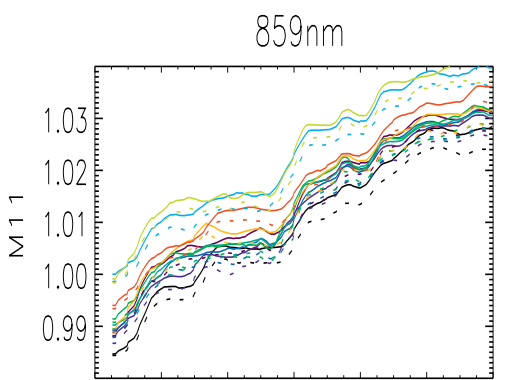

2002200420062008201020122014

Figure 9. $\mathrm{M}_{11}$ for mirror sides 1 (solid line) and 2 (dashed line), detectors 1-10 (black to red) of MODIS Terra as a function of time for all wavelengths at the end of scan scan (frame 1250). 


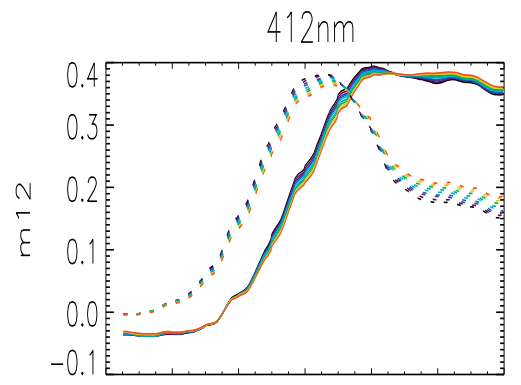

2002200420062008201020122014

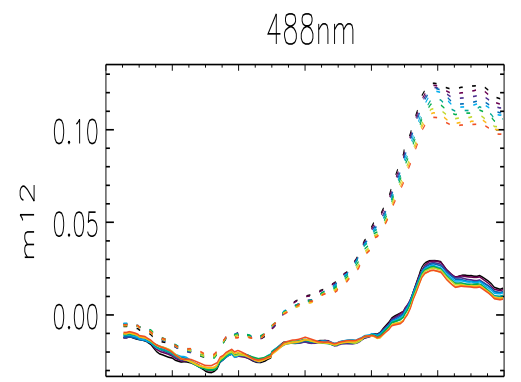

2002200420062008201020122014

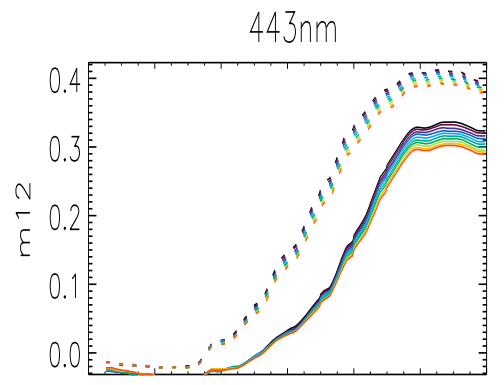

2002200420062008201020122014

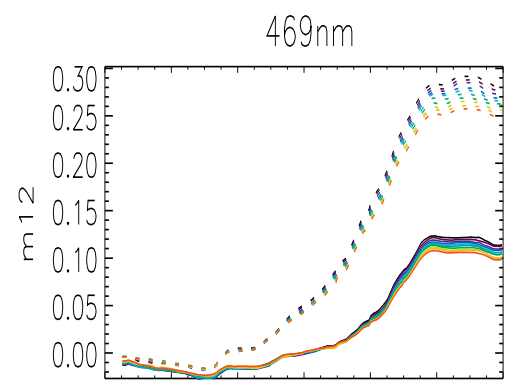

2002200420062008201020122014

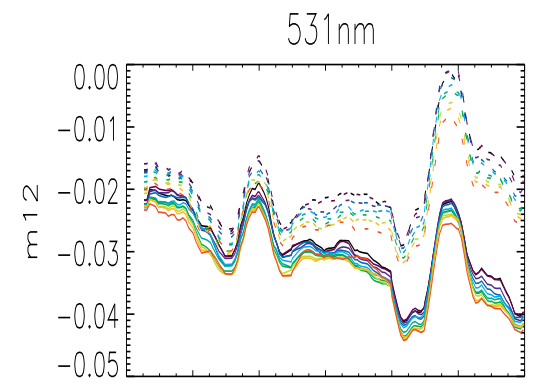

2002200420062008201020122014

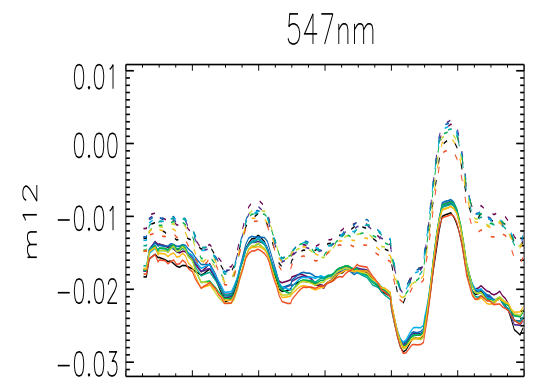

2002200420062008201020122014

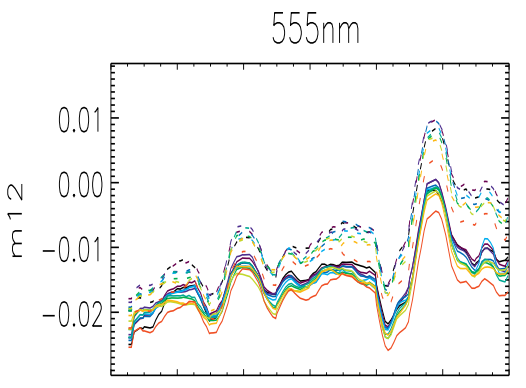

2002200420062008201020122014

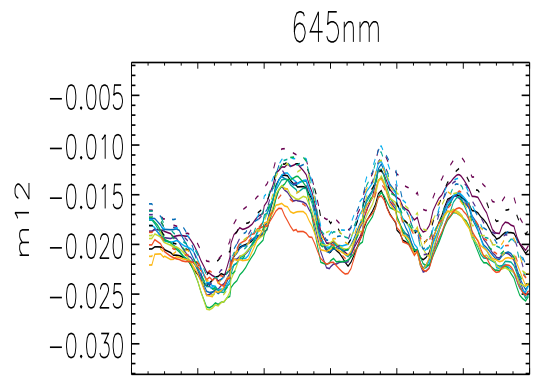

2002200420062008201020122014

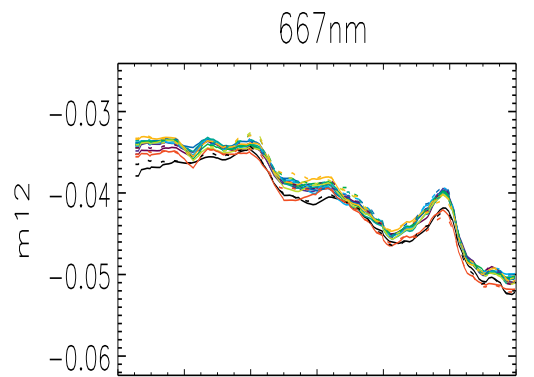

2002200420062008201020122014

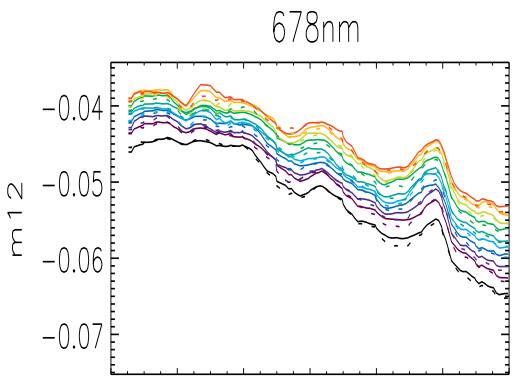

2002200420062008201020122014

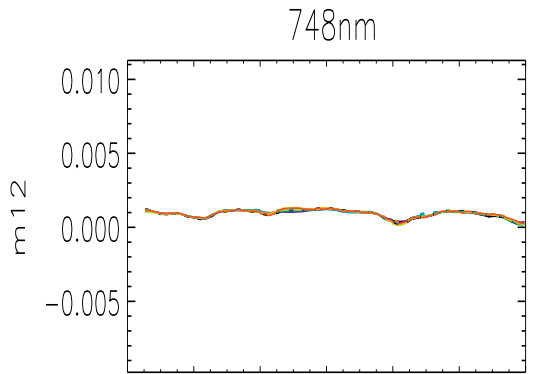

2002200420062008201020122014

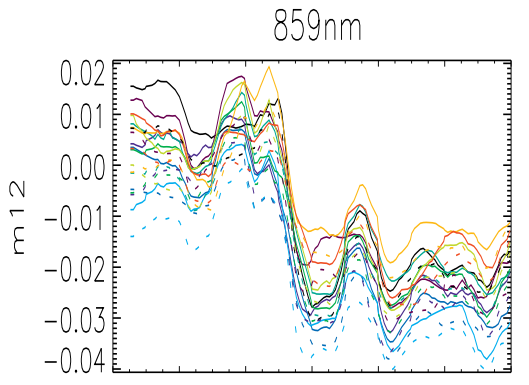

2002200420062008201020122014

Figure 10. $\mathrm{m}_{12}$ for mirror sides 1 (solid line) and 2 (dashed line), detectors 1-10 (black to red) of MODIS Terra as a function of time for all wavelengths at the end of scan scan (frame 1250). 


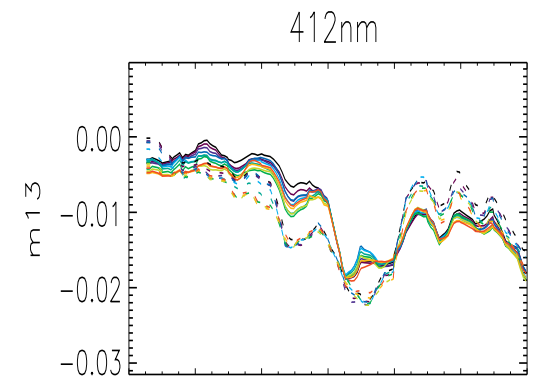

2002200420062008201020122014

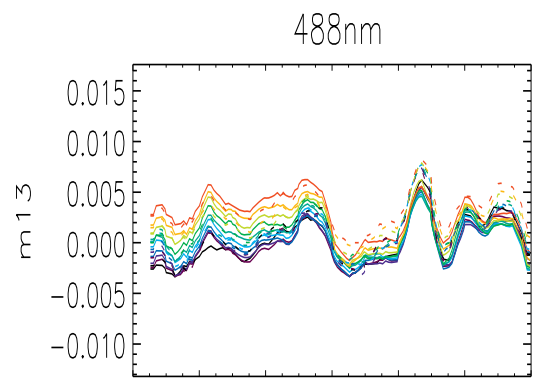

2002200420062008201020122014

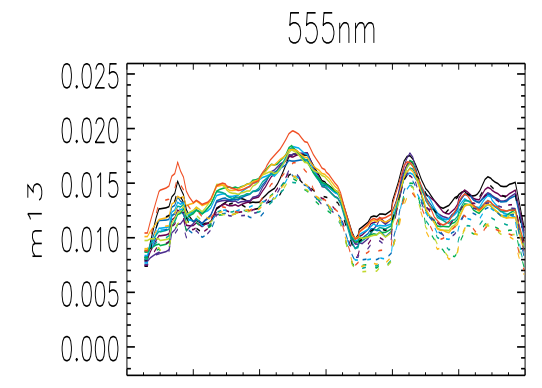

2002200420062008201020122014

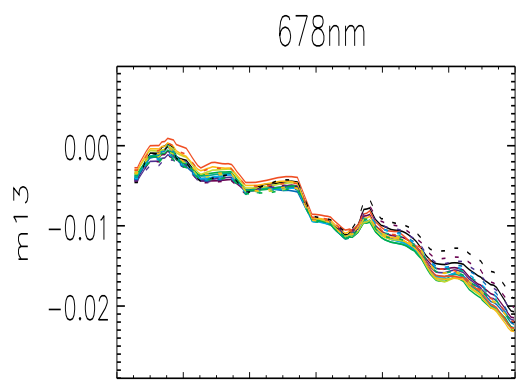

2002200420062008201020122014

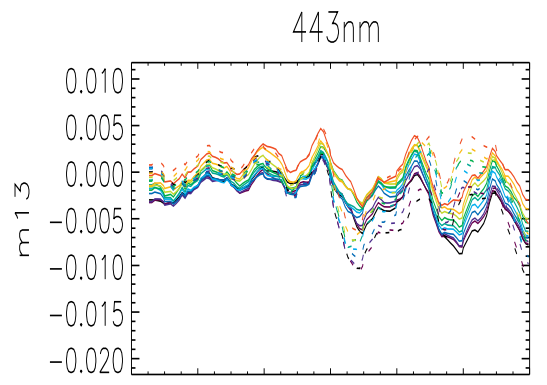

2002200420062008201020122014

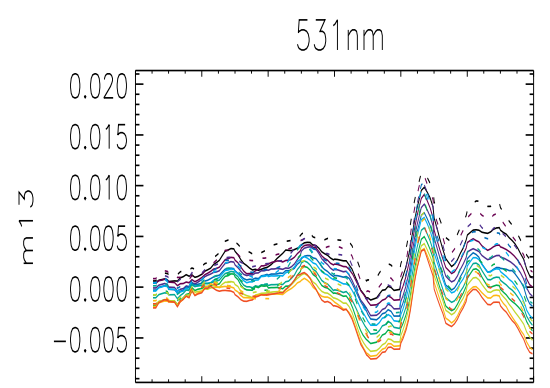

2002200420062008201020122014

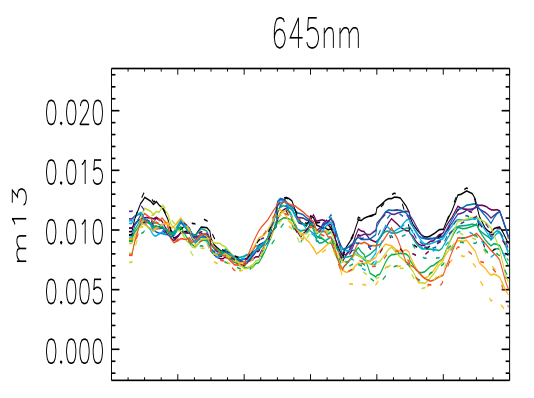

2002200420062008201020122014

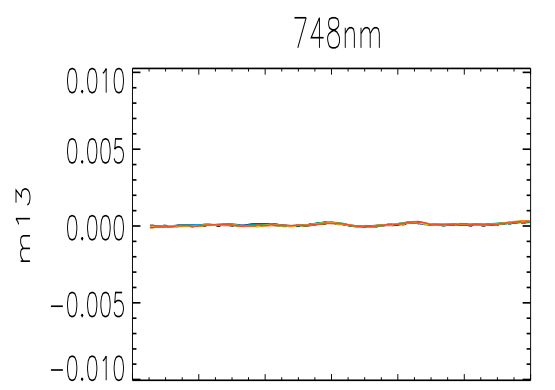

2002200420062008201020122014
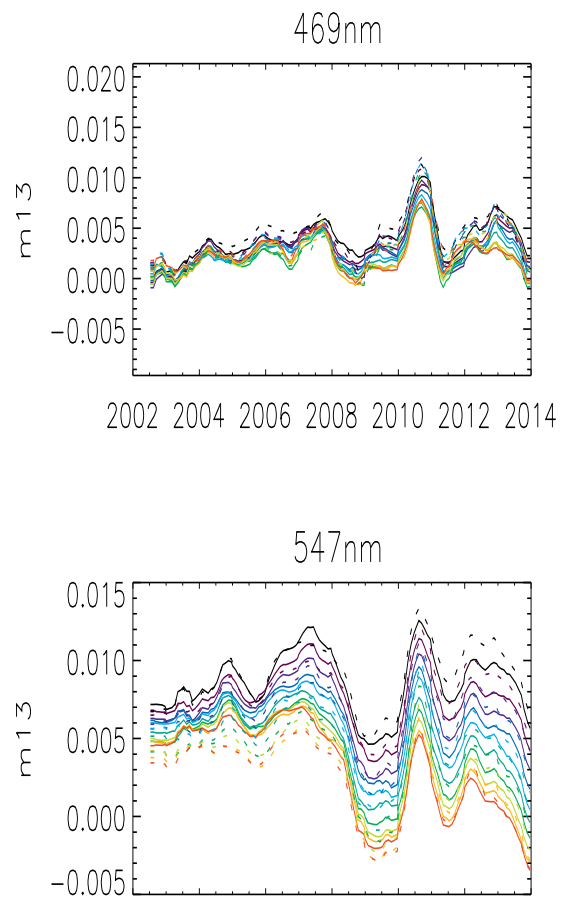

2002200420062008201020122014

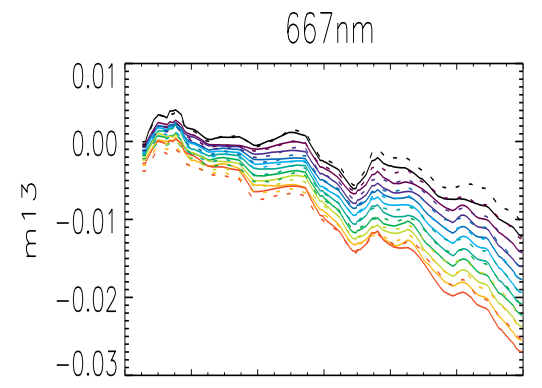

2002200420062008201020122014

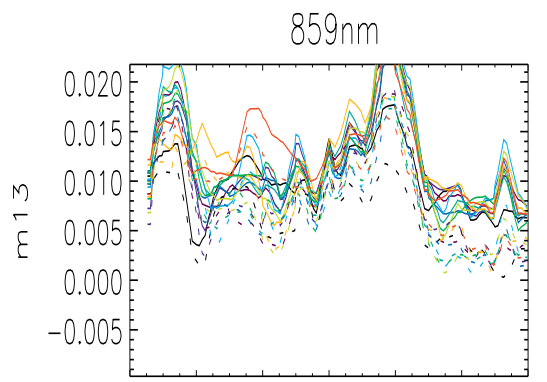

2002200420062008201020122014

Figure 11. $\mathrm{m}_{13}$ for mirror sides 1 (solid line) and 2 (dashed line), detectors 1-10 (black to red) of MODIS Terra as a function of time for all wavelengths at the end of scan scan (frame 1250). 
The functional form of the scan angle dependence for the polarization correction parameters $m_{12}$ and $m_{13}$ was changed from linear ${ }^{6,7}$ to quadratic. This change eliminated an out-of-family behavior of the $m_{12}$ coefficients of band $8(412 \mathrm{~nm})$ at the beginning of the scan in the later half of the Terra mission and should reduce a potential overcorrection reported in the application of the polarization correction coefficients to MODIS Terra land products. ${ }^{15}$

For most bands, the polarization sensitivity increases with time. A notable exception is band 8, mirror side 2 , where the polarization correction parameter $m_{12}$ increased up to 2009 and then fell in the following years to less than half its maximum value. This rather surprising temporal evolution corresponds to an unusual temporal trend of the response versus scan for band 8 mirror side 2 (not shown here).

A comparison of the cross calibration approach retrieving both gain corrections and polarization correction parameters versus retrieving the gain corrections only showed that not including the polarization correction parameters resulted in a large increase of the seasonal oscillation in the retrieved gain corrections for band 8 and in significant differences in the long term trends as well. This underscores the importance of deriving and applying both gain corrections and polarization correction parameters simultaneously.

\section{ACKNOWLEDGMENTS}

We want to thank our colleagues from OBPG and the MODIS Characterization Support Team for their support. This work was funded by the NASA MODIS Science Team.

\section{REFERENCES}

[1] Barnes, W. L., Pagano, T. S., and Salomonson, V. V., "Prelaunch characteristics of the moderate resolution imaging spectroradiometer (MODIS) on EOS-AM1," IEEE Transactions on Geoscience and Remote Sensing 36(4), 1088-1100 (1998).

[2] Franz, B. A., Werdell, P. J., Meister, G., Bailey, S. W., Eplee, R. E., Feldman, G. C., Kwiatkowska, E., McClain, C. R., Patt, F. S., and Thomas, D., "The continuity of ocean color measurements from SeaWiFS to MODIS," Proc. SPIE 5882(1), 58820W, SPIE (2005).

[3] Xiong, X. and Barnes, W. L., "An overview of MODIS radiometric calibration and characterization," $A d-$ vances in Atmospheric Sciences 23(1), 69-79 (2006).

[4] Sun, J., Xiong, X., Barnes, W., and Guenther, B., "MODIS reflective solar bands on-orbit lunar calibration," IEEE Transactions on Geoscience and Remote Sensing 45(7), 2383-2393 (2007).

[5] Franz, B. A., Kwiatkowska, E. J., Meister, G., and McClain, C. R., "Moderate Resolution Imaging Spectroradiometer on Terra: limitations for ocean color applications," Journal of Applied Remote Sensing 2, 023525 (2008).

[6] Kwiatkowska, E. J., Franz, B. A., Meister, G., McClain, C. R., and Xiong, X., "Cross calibration of ocean-color bands from Moderate-Resolution Imaging Spectroradiometer on Terra platform," Applied Optics 47(36), 6796-6810 (2008).

[7] Meister, G. and Franz, B. A., "Adjustments to the MODIS terra radiometric calibration and polarization sensitivity in the 2010 reprocessing," in [Earth Observing Systems XVI], Butler, J. J. and Xiong, J., eds. (2011).

[8] Eplee, R. E., Meister, G., Patt, F. S., Barnes, R. A., Bailey, S. W., Franz, B. A., and McClain, C. R., "On-orbit calibration of SeaWiFS," Applied Optics 51(36), 8702-8730 (2012).

[9] Xiong, X., Sun, J., Xie, X., Barnes, W. L., and Salomonson, V. V., "On-orbit calibration and performance of Aqua MODIS reflective solar bands," IEEE Transactions on Geoscience and Remote Sensing 48(1), 535-546 (2010).

[10] Sun, J., Xiong, X., Angal, A., Chen, H., Wu, A., and Geng, X., "Time-dependent response versus scan angle for MODIS reflective solar bands," IEEE Transactions on Geoscience and Remote Sensing 52(6), 3159-3174 (2014).

[11] Gordon, H. R., Du, T., and Zhang, T., "Atmospheric correction of ocean color sensors: analysis of the effects of residual instrument polarization sensitivity," Applied Optics 36(27), 6938-6948 (1997). 
[12] Sun, J. and Xiong, X., "MODIS polarization sensitivity analysis," IEEE Transactions on Geoscience and Remote Sensing 45(9), 2875-2885 (2007).

[13] Meister, G., Kwiatkowska, E. J., Franz, B. A., Patt, F. S., Feldman, G. C., and McClain, C. R., "ModerateResolution Imaging Spectroradiometer ocean color polarization correction," Applied Optics 44(26), 55245535 (2005).

[14] Franz, B. A., Bailey, S. W., Werdell, P. J., and McClain, C. R., "Sensor-independent approach to the vicarious calibration of satellite ocean color radiometry," Applied Optics 46, 5068-5082 (August 2007).

[15] Alexei Lyapustin, "Science impact of MODIS calibration degradation and C6+ improvements." http://modis.gsfc.nasa.gov/sci_team/meetings/201404/presentations/plenary/lyapustin.pptx (2014).

[16] Eric Vermote, "Surface reflectance over land." http://modis.gsfc.nasa.gov/sci_team/meetings/201404/presentations/plenary/vermote.pdf (2014).

[17] Jeong, M., Hsu, N. C., Kwiatkowska, E. J., Franz, B. A., Meister, G., and Salustro, C. E., "Impacts of cross-platform vicarious calibration on the Deep Blue aerosol retrievals for Moderate Resolution Imaging Spectroradiometer aboard Terra," IEEE Transactions on Geoscience and Remote Sensing, accepted (2011). 